\title{
Real-time detection of faecally contaminated drinking water with tryptophan-like fluorescence: defining threshold values
}

James P.R. Sorensen ${ }^{\mathrm{a}}$, Andy Baker ${ }^{\mathrm{b}}$, Susan A. Cumberland ${ }^{\mathrm{c}}$, Dan J. Lapworth ${ }^{\mathrm{a}}$, Alan M. MacDonald ${ }^{\mathrm{d}}$, Steve Pedley ${ }^{\mathrm{e}}$, Richard G. Taylor ${ }^{\mathrm{f}}$, Jade S.T. Ward ${ }^{\mathrm{a}, \mathrm{e}}$

${ }^{a}$ British Geological Survey, Maclean Building, Wallingford, OX10 8BB, UK

${ }^{\mathrm{b}}$ Connected Waters Initiative Research Centre, UNSW Australia, Sydney, New South Wales 2052, Australia

${ }^{\mathrm{c}}$ Australian Synchrotron, Clayton, Victoria 3168, Australia

${ }^{d}$ British Geological Survey, Lyell Centre, Edinburgh EH14 4AP, UK

${ }^{\mathrm{e}}$ Department of Civil and Environmental Engineering, University of Surrey, Guildford, GU2 $7 \mathrm{XH}, \mathrm{UK}$

${ }^{\mathrm{f}}$ Department of Geography, University College London, London WC1E 6BT, UK

Keywords: tryptophan-like fluorescence; fluorescence spectroscopy; faecal contamination; drinking water; thermotolerant (faecal) coliforms

* Corresponding author: James Sorensen jare1@bgs.ac.uk

\section{Abstract}

We assess the use of fluorescent dissolved organic matter at excitation-emission wavelengths of $280 \mathrm{~nm}$ and $360 \mathrm{~nm}$, termed tryptophan-like fluorescence (TLF), as an indicator of faecally contaminated drinking water. A significant logistic regression model was developed using TLF as a predictor of thermotolerant coliforms (TTCs) using data from groundwater- and surface water-derived drinking water sources in India, Malawi, South Africa and Zambia. A TLF threshold of $1.3 \mathrm{ppb}$ dissolved tryptophan was selected to classify TTC contamination. Validation of the TLF threshold indicated a false-negative error rate of $15 \%$ and a false-positive error rate of $18 \%$. The threshold was unsuccessful at classifying contaminated sources containing $<10$ TTC cfu per $100 \mathrm{~mL}$, which we consider the current limit of detection. If only sources above this limit were classified, the false-negative error rate was very low at $4 \%$. TLF intensity was very strongly correlated with TTC concentration $\left(\rho_{\mathrm{s}}=0.80\right)$. A higher threshold 
of $6.9 \mathrm{ppb}$ dissolved tryptophan is proposed to indicate heavily contaminated sources (>100 TTC cfu per $100 \mathrm{~mL}$ ). Current commercially available fluorimeters are easy-to-use, suitable for use online and in remote environments, require neither reagents nor consumables, and crucially provide an instantaneous reading. TLF measurements are not appreciably impaired by common intereferents, such as $\mathrm{pH}$, turbidity and temperature, within typical natural ranges. The technology is a viable option for the real-time screening of faecally contaminated drinking water globally.

\section{Introduction}

Drinking water contaminated with faeces is consumed by an estimated 1.8 billion people globally (Bain et al. 2014a). This constitutes a major burden on public health due to the ingestion of enteric pathogens that cause infectious diseases (Bain et al. 2014b). Most significantly, it is considered to result in more than half a million deaths per annum from diarrhoea alone, with children under five particularly at risk (Prüss-Ustün et al. 2014). Accordingly, the United Nations have established Sustainable Development Goals (SDGs) for the universal access to safe drinking water for all and improvements in water quality by 2030 .

Assessment of the faecal contamination of drinking water is typically inferred through the presence of surrogate indicator organisms, such as thermotolerant coliforms (TTCs), including Escherichia coli (WHO 2011). Such bacteriological analyses requires well-trained personnel, working with sterile equipment and reagents, and entails $>18 \mathrm{~h}$ work due to the necessity for culturing. In lower- and middle-income countries, these requirements can restrict: on-site bacteriological testing of water, the understanding and communication of risks to users, and subsequently behavioural change in communities (UNICEF 2017), i.e. limiting progress towards meeting the SDGs. These methodological requirements even limit the ability of water companies in the most developed countries from assessing bacteriological water quality 
beyond, typically, a daily basis. Consequently, poor bacteriological quality drinking water can be released into a municipal supply network between testing and sporadic disease outbreaks still occur (Adler et al. 2017). Therefore, there is a global need for an easy-to-use technology that can detect faecal contamination in drinking water in real-time.

Numerous researchers have already proposed alternative methods (Berg and Fiksdal 1988, Chen et al. 2015, Frahm and Obst 2003, Guion et al. 2008, Gunda et al. 2014, Harwood et al. 2014, Lopez-Roldan et al. 2013, Maheux et al. 2011, Radke and Alocilja 2005, Rinttilä et al. 2004, Rompré et al. 2002, Velasquez-Orta et al. 2017), yet this has not led to a single successful commercial product. Tryptophan-like fluorescence (TLF) is a component of UV-fluorescent dissolved organic matter at excitation-emission wavelengths of $280 \mathrm{~nm}$ and $360 \mathrm{~nm}$ and is a viable potential alternative. The term TLF is used to reflect the array of generally aromatic and proteinaceous compounds that share similar fluorescence properties to the amino acid tryptophan. It has long been considered an indicator of wastewater and biological activity in freshwater environments (Baker 2001, Cammack et al. 2004, Carstea et al. 2016) and this has led to increased interest in its use as an early indicator of drinking water quality (Baker et al. 2015, Heibati et al. 2017, Sorensen et al. 2015a, Sorensen et al. 2015b, Sorensen et al. 2016, Stedmon et al. 2011). Crucially, TLF can now be quantified instantaneously in the field using commercially available LED UV-based fluorimeters that express TLF intensity as an equivalent concentration of dissolved tryptophan in parts per billion.

E. coli cells have been proven to directly emit TLF and also excrete compounds that fluoresce in the TLF region in controlled laboratory studies (Bronk and Reinisch 1993, Dalterio et al. 1986, Dalterio et al. 1987, Dartnell et al. 2013, Fox et al. 2017, Seaver et al. 1998). For example, Fox et al. (2017) demonstrated a strong correlation $\left(\mathrm{r}^{2}=0.98\right)$ between TLF intensity and $E$. coli concentration during culturing, with the majority of TLF being intracellular in origin. 
Notably, E. coli is the preferred organism for the industrial production of tryptophan by fermentation of carbohydrates (Ikeda 2006). Alternatively, if tryptophan is readily available in the environment, E. coli will import and hydrolyse tryptophan, almost quantitatively into indole that is then excreted (Li and Young 2013). This would also enhance any TLF signal because pure indole fluoresces within the TLF region at 33\% greater intensity than tryptophan (Figure 1). It has also been observed that $E$. coli excrete tryptophan under nutrient limited conditions as they transit from a culturable to a dormant viable but non-culturable state (Arana et al. 2004). This will eventually occur when E. coli are released into many freshwater environments, and particularly groundwater where nutrients are typically limited.

In recent field studies of drinking water quality using commercially available portable fluorimeters, it has been demonstrated that TLF is significantly more intense in groundwaterderived sources contaminated with TTCs than those where TTCs were absent (Sorensen et al. 2015a, Sorensen et al. 2016). Moreover, significant positive correlations between TLF intensity and TTC concentration have also been observed in both groundwater- and surface waterderived sources (Baker et al. 2015, Sorensen et al. 2015a, Sorensen et al. 2016), in addition to TLF being elevated in the presence of enteric pathogens (Sorensen et al. 2015b). We now evaluate the ability of TLF to detect faecally contaminated drinking water against an assessment criteria that includes sensor performance, design, and interference. To evaluate sensor performance a global dataset will be used to define optimal TLF thresholds to determine faecal contamination that are universally applicable.

\section{Methods}

\subsection{Available TLF-TTC data}

Concurrent TLF and TTC data were collated from a mixture of unpublished and published studies of drinking water in four separate countries: India, Malawi, South Africa and Zambia 
(Table 1). The Indian study was conducted in Bihar State and 150 groundwater sources were selected to achieve spatial coverage across four villages with an approximate split between those near $(<10 \mathrm{~m})$ and those away $(>10 \mathrm{~m})$ from recently installed on-site sanitation (Sorensen et al. 2016). The Malawian study predominantly comprised sampling a randomly selected groundwater source in 40 randomly selected villages in each of five districts of the country (Balaka, Machinga, Lilongwe, Nkhotakota, Mzimba). In total, 39 of the 200 randomly selected sources were non-functional and an additional 21 sources were opportunistically sampled near the original randomly selected source. The South African study involved repeated sampling of 28 locations selected upstream, near to, and downstream of expected contributing sources of poor water quality in two surface water catchments in KwaZulu Natal (Baker et al. 2015). The Zambian study comprised sampling 65 groundwater sources across peri-urban, industrial, and lower and higher income residential land uses in the city of Kabwe, of which 46 sources were sampled during both wet and dry seasons (Sorensen et al. 2015a).

All studies used a portable fluorimeter targeting TLF on an unfiltered water sample and enumerated either TTCs by membrane filtration or E. coli by the Colilert ${ }^{\circledR}$ (IDEXX) method (Table 1). Although the studies employed differing faecal indicator organisms, Hamilton et al. (2005) noted that E. coli by the Colilert method accounted for $104 \%$ of TTCs enumerated by membrane filtration in temperate surface waters. In tropical groundwaters, impacted by inadequate sanitation, 90-99\% of all TTCs have been shown to be E. coli (Howard et al. 2003, Leclerc et al. 2001). Therefore we consider the number of faecal indicator organisms to be comparable between studies. Furthermore, relationships between TLF and the concentration of indicator organisms will be addressed using WHO risk categories (1997) that are classified based on a logarithmic scale of faecal indicator organisms that does not distinguish between TTCs and E. coli. 


\subsection{Assessment criteria}

The ability of TLF to detect faecally contaminated drinking water was assessed using the UNICEF target product profile for a new method for the more rapid detection of $E$. coli (Table 2) (UNICEF 2017). This incorporates sensor performance, design and interferences. As a minimum, any new methodology should be able to determine faecal contamination within $3 \mathrm{~h}$ with a $10 \%$ false-positive and false-negative error rate, have a limit of detection of $10 \mathrm{cfu} / 100 \mathrm{~mL}$, and quantify faecal contamination according to the WHO risk categories.(1997) Additionally, the method should be easy-to-use, suitable for fieldwork in remote locations, cost $<\$ 5$ per test over its lifetime, and not be adversely affected by a range of common interferents such as turbidity and $\mathrm{pH}$.

\subsection{Determining faecal contamination}

The extent to which TLF indicated the presence of TTCs, the indicator of faecal contamination, was investigated using a logistic regression model (Dobson 2001) in R software version 3.2.2. The model was developed using a training dataset, then evaluated against a validation dataset. To produce these datasets, firstly, the collated dataset $(n=564)$ was grouped into WHO risk categories (WHO 1997). These risk categories are based on the number of faecal indicator organisms per $100 \mathrm{~mL}$ : Very Low risk (0), Low risk (1-9), Intermediate risk (10-99), High risk (100-999), and Very High risk (1000+). Subsequently, the data were randomly sampled to split the data equally in each risk category using the R package 'caret'. This produced training and validation datasets for each risk category, which were combined to form collated training $(\mathrm{n}=286)$ and validation datasets $(\mathrm{n}=276)$. 
The performance of the model was initially assessed using the receiver-operating characteristic (ROC) curve (Hanley and McNeil 1983) implemented in the R package 'pROC' (Robin et al. 2011). The ROC curve is a plot of the false-positive rate (FPR - the proportion of uncontaminated sites falsely classified as contaminated) against the true-positive rate (TPR the proportion of contaminated sites correctly classified) at varying TLF thresholds. The area under this curve (AUC) is a measure of the effectiveness of the classifier: it will be 1 for a perfect classifier and 0.5 if the classifier is performing no better than a random choice.

An optimal TLF threshold was selected as the value that provides the lowest sum of the FPR and the false-negative rate (FNR - the proportion of contaminated sites incorrectly classified as uncontaminated) for samples in the Very Low to Medium training risk categories. These lower risk categories were used to select the TLF threshold to ensure it was optimised around the limits of detection outlined in the assessment criteria. This threshold was then used to classify the validation dataset as a whole, then each validation risk category dataset individually. The threshold performance was assessed in terms of FPR and FNR for consistency with the assessment criteria. Finally, the threshold was used to classify data from the individual studies separately to evaluate any potential discrepancies.

\subsection{Quantifying faecal contamination}

There is a significant very strong positive correlation between TLF intensity and TTC concentration in the collated dataset $\left(\rho_{\mathrm{s}}=0.80, \mathrm{p}<0.001\right)$, but due to the amount of scatter it was considered unlikely that TLF could predict an equivalent plate count. Hence, the ability of TLF to distinguish between WHO risk categories was investigated instead. Consequently, the TLF data were grouped into the risk categories and the non-parametric Kruskal-Wallis test was performed. A non-parametric pairwise multiple comparison was then conducted using Dunn's Method to assess differences between all individual categories. We then attempted to define 
TLF threshold values for each individual WHO risk category using the same training and validation datasets acquired using methods outlined in section 2.3. TLF thresholds were defined for each risk category by selecting the mid-point between the upper and lower quartiles of adjacent risk categories in the training dataset and then evaluated by classifying the validation datasets. These thresholds were not tested against individual studies because there were insufficient data across the risk categories to facilitate evaluation.

\section{Results and discussion}

\subsection{Determining faecal contamination and limit of detection}

Tryptophan-like fluorescence is a significant predictor of the presence of TTCs for the training dataset according to the logistic regression model $(p<0.001)$. The area under the ROC curve was 0.90 (Figure 2a), which is much closer to the perfect classifier of 1 than the random selection value of 0.5 . The optimal selected TLF threshold value of $1.3 \mathrm{ppb}$ resulted in a FNR and FPR of $14 \%$ and $21 \%$, respectively (Figure 2b). The overall FNR is slightly high, but it is biased by the Low risk category where the classification is unsuccessful (52\%). Omitting this category the overall FNR reduces to within the ideal performance of 5\% (5\%). The Medium risk category data are classified to close to the minimum assessment criterion (12\%), whilst the High and Very High risk datasets are successfully classified with FNRs of 9 and $0 \%$, respectively (Figure 3).

The TLF threshold performs similarly classifying the validation dataset with an overall FNR and FPR of 15\% and 18\%, respectively. Again, the threshold is unsuccessful at classifying sites in the Low risk category, with a FNR of 50\%, which may, in part, result from limited reproducibility in the TTC counts themselves at low numbers. If we exclude these Low risk data then the overall FNR reduces to within the ideal criterion of 5\% (4\%). The individual 
Medium, High, and Very High validation datasets are classified close to or within the minimum assessment criterion with FNRs of 12\%, 3\% and 2\%, respectively (Figure 3).

Given the inability of the threshold to classify Low risk sites, we deem the limit of detection is currently Medium risk, i.e. at least $10 \mathrm{cfu} / 100 \mathrm{~mL}$. To refine a precise detection limit requires considerably more data around $10 \mathrm{cfu} / 100 \mathrm{~mL}$. This detection limit should be considered sufficient given any relationship between $E$. coli concentration in drinking water and diarrhoea is frequently only significant above at least $10 \mathrm{cfu} / 100 \mathrm{~mL}$ (Brown et al. 2008, Moe et al. 1991).

The TLF threshold of $1.3 \mathrm{ppb}$ is generally effective at classifying data from individual studies (Figure 4). TTC negative data in the Indian study are classified within the assessment criteria with a FPR of only $9 \%$. However, FPRs for these uncontaminated sites are high in both the Malawian and Zambian datasets. The elevated FPR of 35\% in the Zambian study combined with the near absence of false-negatives suggests a higher TLF threshold may be warranted for a more equal distribution of errors. However, a logistic regression model developed solely using the Very Low, Low and Medium risk Zambian datasets indicates only a marginally higher optimal threshold of $1.6 \mathrm{ppb}$. Here, the FPR still remains too high (24\%) and the FNR is acceptable (7\%). The majority of unsuccessfully classified Low risk data are from the Indian and Malawian datasets. Where error rates exceed the $10 \%$ minimum criterion for Medium and High risk categories in individual studies, it is a result of only one or two incorrectly classified sources suggesting that sample sizes are too small to robustly estimate error rates for these categories. All Very High risk data are successfully classified with a FNR of only $0-1 \%$ in all studies.

\subsection{Quantifying faecal contamination}

There are significant differences in median TLF between risk categories indicating that multiple populations exist in the dataset (Kruskal-Wallis, $p<0.001$ ). Significant differences in 
median TLF exist between all risk categories (Figure 5), with the exception of Medium and High (Dunn's Method, $p=0.96$ ). The TLF thresholds, defined in Table 3, have error rates that are too high for practical implementation when assessed against the validation dataset (Figure 6). Nevertheless, incorrectly classified sites are most likely to fall in the immediately adjacent risk categories. For example, $90 \%$ of Very Low risk sites are classified as Very Low to Low risk and $91 \%$ of Very High risk sites are classified as High to Very High risk.

Therefore, we propose a threshold of $1.3 \mathrm{ppb}$ for classifying at-risk sources $(>10 \mathrm{cfu} / 100 \mathrm{~mL})$ according to the logistic regression model in Section 3.1. This would effectively classify sites in the validation dataset according to Figure 3 with a FNR of only $4 \%$ for sources that are at least Medium risk. It would be unsuccessful at classifying Low risk sites (FPR $=50 \%)$ and reasonably successful at classifying Very Low risk sites $(\mathrm{FPR}=21 \%)$.

A greater threshold of $6.9 \mathrm{ppb}$ is proposed for classifying higher risk sources $(>100 \mathrm{cfu} / 100 \mathrm{~mL})$ following the TLF thresholds defined in Table 3 . This threshold successfully classifies sources in the validation dataset that are at least High risk with a FNR of $17 \%$ and incorrectly classifies lower risk sites with a FPR of $4 \%$. However, due the TLF overlap between Medium and High risk categories (Figure 5) the threshold produces a FPR for Medium risk sites of $31 \%$ and a FNR for High risk sites of $30 \%$. Notwithstanding, at such an elevated TLF the FNR for Very High risk sources remains low (9\%) and the FPR for Very Low and Low risk sites is very low $(2 \%)$.

\subsection{Sensor designs}

TLF can simply be measured instantaneously in a covered beaker or cuvette using a fluorimeter (Figure 7), with no need for reagents, consumables or incubation. There are currently several commercially available battery-powered fluorimeters on the market that are suitable for fieldwork in remote locations, although these vary in design and method. They include 
submersible sensors, e.g. UviLux (Chelsea Technologies Group Ltd, UK) and Cyclops-7 ${ }^{\mathrm{TM}}$ (Turner Designs, USA), or cuvette-based systems, e.g. SMF4 (STS Instrument Ltd, UK). Cuvette-based systems are advantageous because they require very small volumes of water $(<5 \mathrm{~mL})$ and contamination of the sample is less likely. This advantage arises because the housing of submersible sensors comes into contact with the sample and the housing can be easily manually handled and can also begin to degrade allowing suspended particles to collect in surface imperfections and potentially leach residue TLF into subsequent samples. This limitation could partially be overcome by mounting submersible sensors in flow-through cells. In addition, it is notable that submersible sensors can produce an apparently very low TLF intensity when air is trapped adjacent to sensor windows. The main advantage of submersible sensors is for online deployments in a flow-cell or directly within a water body.

The most critical element to currently consider when selecting a sensor is currently the limit of detection and not whether it is submersible or cuvette-based. The proposed $1.3 \mathrm{ppb}$ threshold for the detection of faecal contamination is below the specification for the majority of sensors on the market. For example, Khamis et al. (2015) demonstrated a minimum detection limit (MDL) of $1.99 \pm 0.53 \mathrm{ppb}$ for the Cyclops $-7^{\mathrm{TM}}$ and Khamis et al. (2016) reported a MDL of $1.74 \mathrm{ppb}$ for a modified submersible GGUN-FL30 (Albillia Co, Switzerland). The only commercially available field fluorimeter we are aware of that is capable of sufficient sensitivity is the submersible UviLux fluorimeter, which incorporates a photomultiplier to amplify the signal and achieve a MDL of up to $0.17 \pm 0.06 \mathrm{ppb}$.(Khamis et al. 2015)

The present generation of field fluorimeters cost in the region of $\$ 5,000-6,000$. This excludes any accessories, such as handheld readers or cables, which can inflate costs by a further $\$ 2,000$ 3,000. Nevertheless, once procured there are no ongoing consumable costs. The expected lifetime of these fluorimeters is estimated to be at least 10 years; hence the costs would be 
within the minimum criterion of $\$ 5$ if used at only 100 sites a year. The sensor lifetime is determined by the LEDs and light filters. To account for a loss of output from the LED and change in the filters over time, it is recommended to undertake a calibration check annually with dissolved tryptophan standards, which can be done by the end user. Additionally, regular negative controls should also be performed using high quality deionised water. Ideally manufacturers would supply sealed long-life containers for both calibration and negative control, but these are currently only available for cuvette-based instruments. Current cuvettes for calibration contain another fluorescent compound, quinine sulphate, which does not fluoresce directly within the TLF region. High concentration standards can be used to assess drift, but these do not reflect the range of TLF intensity encountered in the environment and these standards cannot be used for a robust calibration. In conclusion, current commercially available fluorimeters adhere to all the assessment criteria concerning sensor design.

\subsection{Potential interferents}

There are a range of matrix interferences that can impact on TLF, including $\mathrm{pH}$, temperature, and turbidity (Hudson et al. 2007, Khamis et al. 2015), but these have not been considered to have substantially affected previous TLF-TTC studies (Baker et al. 2015, Sorensen et al. 2015a, Sorensen et al. 2016). Reynolds et al. (2003) documents a $\pm 3 \%$ variation in TLF from pure dissolved tryptophan when the sample matrix was modified between $\mathrm{pH} 5$ and 8 . Baker et al. (2007) observed an order of magnitude greater quenching in TLF for urban surface water samples for a $\mathrm{pH}$ range of 5 to 9 .

Khamis et al. (2015) noted that TLF emitted from dissolved tryptophan standards was not attenuated by the addition of particles treated with hydrogen peroxide, to remove organic matter, below 50 NTU turbidity. Instead, suspended particles scattered the emitted light and increased the intensity reaching the detector. Larger silt particles were more efficient than 
smaller clay particles at scattering light. However, multiple high turbidity ( $>10$ NTU) groundwater sites in India and Malawi contained low TLF $(<1 \mathrm{ppb})$ suggesting that the scattering of light may be insignificant in natural settings. The issue of turbidity could be partially addressed by widening the separation between the excitation and emission wavelengths. Currently, sensors have broad filters for both excitation and emission to maximise fluorescent output and detection. For example, the Cyclops $7^{\mathrm{TM}}$ has an excitation output of $285 \pm 10 \mathrm{~nm}$ and emission set at $350 \pm 55 \mathrm{~nm}$, i.e. there is overlap at $295 \mathrm{~nm}$. Low salinity in drinking water is unlikely to have an appreciable impact on TLF. Overall, there is limited evidence, to date, that the range of interferents outlined in the assessment criteria has any substantial adverse impact on the TLF of raw drinking water.

One interferent not considered in the criteria is temperature. Baker (2005) noted quenching of TLF by $20 \pm 4 \%$ to $35 \pm 5 \%$ between 10 and $45^{\circ} \mathrm{C}$ in rivers and wastewaters. Such temperature quenching is generally considered to be linear (Khamis et al. 2015), hence it is possible that sensors could employ automatic corrections for temperature. Researchers have already developed such algorithms and demonstrated their value for in-situ fluorimeter deployments (Khamis et al. 2015, Shutova et al. 2016). However, such studies have explored the temperature quenching of dissolved tryptophan, whilst TLF in field studies is known to quench at different rates in different water types dependent upon its composition (Baker 2005). The thresholds defined in this paper do not consider the influence of temperature, which was broadly similar across all studies: India $\left(24-29^{\circ} \mathrm{C}\right)$, Malawi $\left(21-29^{\circ} \mathrm{C}\right)$, South Africa $\left(20-26^{\circ} \mathrm{C}\right)$, Zambia $(24-$ $\left.28^{\circ} \mathrm{C}\right)$.

There are multiple peaks of fluorescent dissolved organic matter that overlap with the tryptophan-like region and could dominate, or give rise to an apparent, TLF signal. For example, part of the region of humic-like fluorescence can overlap with that of TLF and 
provide an additional baseline component or potentially mask the TLF signal completely. In addition, proteins in organic waste (Muller et al. 2011) and xenobiotic compounds, e.g. polycyclic aromatic hydrocarbons (Baker and Curry 2004) and diesel pollution (Carstea et al. 2010), may directly fluoresce in the TLF region. Such interference that is unrelated to faecal contamination could result in false-positives.

Interference between fluorescent regions is a long-recognised issue for researchers using laboratory-based spectrofluorometers which scan over a large range of excitation-emission wavelengths, but is a real limitation of single-wavelength sensors. Studies using laboratory spectrofluorometers address the issue by investigating ratios of fluorescent peaks (Baker 2002, Lapworth et al. 2008) or by a modelling approach to elucidate individual peaks known as PARAllel FACtor analysis (PARAFAC) (Baghoth et al. 2011). In the field, it is possible to use multiple single-wavelength sensors to evaluate potential interference (Sorensen et al. 2016). Additionally, there is an increasing move towards the development of dual-wavelength pair sensors that can output ratios as well as absolute TLF (Bridgeman et al. 2015, Li et al. 2016).

Other potential interferences include the absorption of light by the sample matrix (the inner filtering effect), quenching by high concentrations of metal ions, or by water treatment processes such as chlorination that is known to quench TLF. See Henderson et al. (2009) for a review of the impacts of water treatment processes on fluorescent dissolved organic matter.

\subsection{Future work}

Currently, false-positive error rates $(18 \%)$ are too high, particularly in specific studies. Further work should better constrain the temporal relationship between TLF and TTCs at individual sources to investigate whether elevated TLF is indicative of sporadic TTC contamination at certain times of the year. The only current evidence for this is in the Zambian study where snap-shot seasonal sampling campaigns of some sources showed perennially elevated TLF 
whilst TTC varied substantially between dry and wet seasons (Sorensen et al. 2015a). If TLF is more resilient than TTCs in water sources, then the use of TLF could be considered advantageous for detecting at-risk sources irrespective of the time of year selected for sampling and given that TTCs are not as long-lived as other pathogens in the environment.(John and Rose 2005)

There is uncertainty over what is actually measured when we quantify TLF in freshwater environments. Compounds emitting TLF can predominantly be: contained within bacterial cells (Determann et al. 1998, Fox et al. 2017), associated with particles (Baker et al. 2007), or entirely freely dissolved (Sorensen et al. 2016, Yamashita and Tanoue 2003); and there remains uncertainty as to the relative contribution from each source. Further, it is questionable whether TLF is a selective indicator of TTCs. There are many other bacteria species that fluoresce in the TLF region because of tryptophan residues in proteins (Bronk and Reinisch 1993, Dalterio et al. 1986, Dalterio et al. 1987, Dartnell et al. 2013, Fox et al. 2017, Seaver et al. 1998, Sohn et al. 2009) and/or excrete compounds that fluoresce in the TLF region, such as Pseudomonas aeruginosa that is ubiquitous in freshwater systems (Elliott et al. 2006, Fox et al. 2017). Therefore, future work should investigate whether TLF is an indicator of total bacteria cells in drinking water as opposed to specifically TTCs.

\section{Conclusions}

A tryptophan-like fluorescence (TLF) threshold equivalent to $1.3 \mathrm{ppb}$ dissolved tryptophan can instantaneously predict the presence of thermotolerant coliforms (TTCs) with a false-positive error rate of $18 \%$ and a false-negative error rate of $15 \%$. However, this TLF threshold is not effective at classifying a contaminated sample with less than $10 \mathrm{TTC}$ cfu/100 mL, which we currently consider to be the limit of detection. If only contaminated sources above this limit of 
detection are classified then the false-negative error rate is very low at $4 \%$. A greater threshold of $6.9 \mathrm{ppb}$ is proposed for classifying higher risk sources (>100 TTC cfu/100 mL).

TLF can be quantified instantaneously using existing commercially available fluorimeters that are battery-powered and handheld for use in remote environments. Its analysis can simply be undertaken by submerging a fluorimeter in a covered container of water. The lifespan of current fluorimeters is anticipated to be in excess of ten years with annual checks and infrequent maintenance. Current procurement costs are in the order of $\$ 5000-6000$ and per test costs would fall below $\$ 5$ within 1000 samples, as there is an absence of ongoing consumable costs. TLF measurements are unlikely to be appreciably impaired by common interferents, such as $\mathrm{pH}$, turbidity, and temperature, within typical natural ranges. Interference from other fluorescent compounds is likely to be more problematic.

The technology should be considered a viable option for the real-time detection of faecally contaminated drinking water which is still consumed by 1.8 billion people globally. It has now been successfully demonstrated in multiple different settings from surface water to groundwater and across varying climatic zones. However, all analysis herein was performed on a modest dataset $(n=564)$, which may contain sampling bias as the majority of data have not been drawn from an independent random sample. There is a need for a large-scale field demonstration of the technique to robustly support the results of this study. 


\section{Figures}

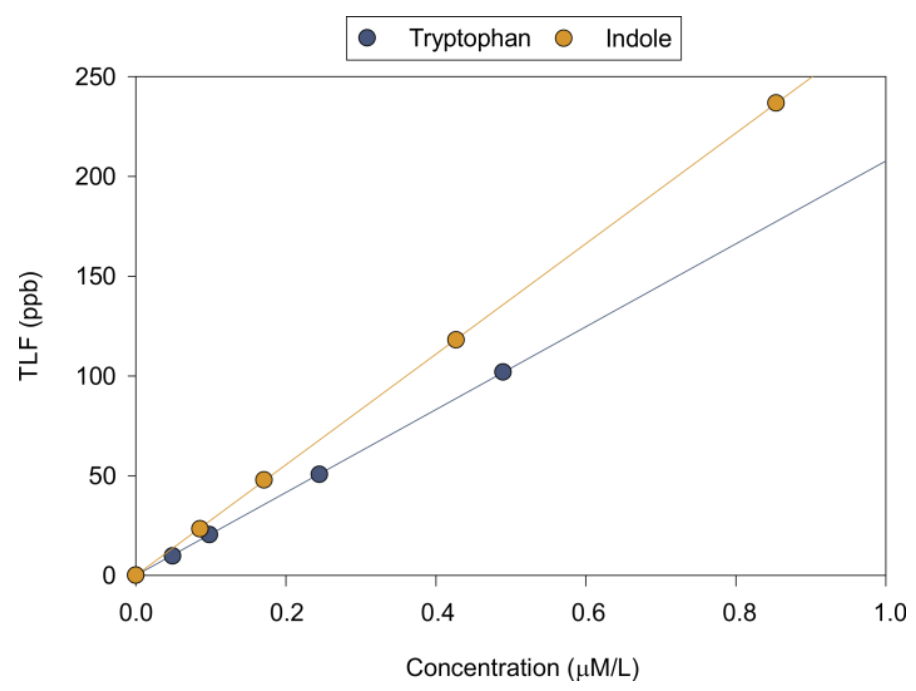

Figure 1. Comparison of the TLF intensity emitted by dissolved tryptophan and indole. Analyses were performed on $0,10,20,50$, and $100 \mathrm{ppb}$ solutions of both compounds with a UviLux fluorimeter (Chelsea Technologies Group Ltd, UK). The gradients of the regression lines are 278 and 209 for indole and tryptophan, respectively.
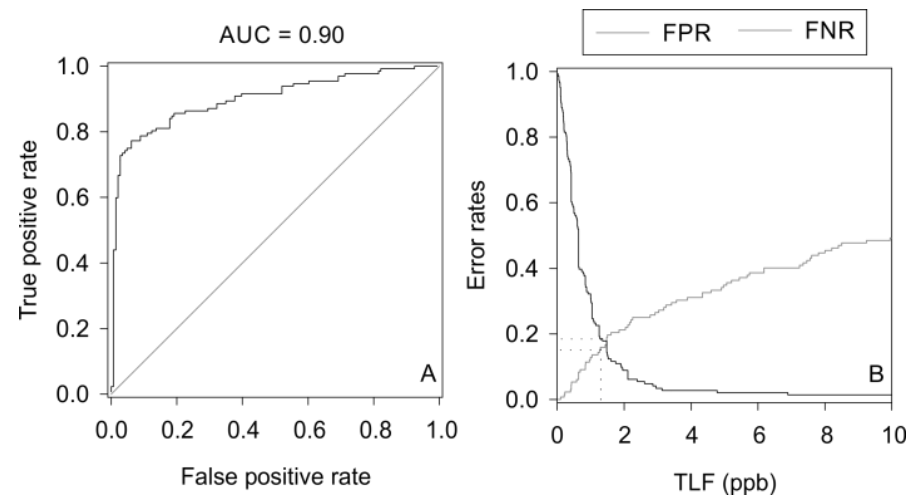

Figure 2. (A) Receiver operator characteristic curve for the classifier of the presence of TTCs using TLF and the performance of a random variable; (B) false-positive (FPR) and falsenegative (FNR) error rates for this classifier. 


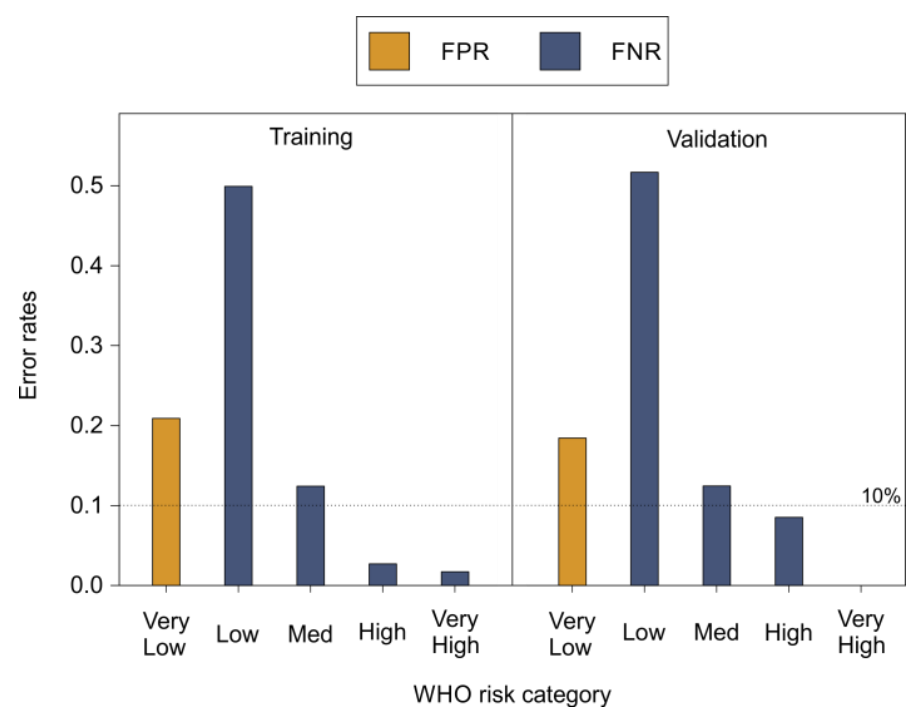

Figure 3. Training and validation false-positive (FPR) and false-negative (FNR) error rates for presence of TTCs using a TLF threshold of $1.3 \mathrm{ppb}$ for each WHO risk category.

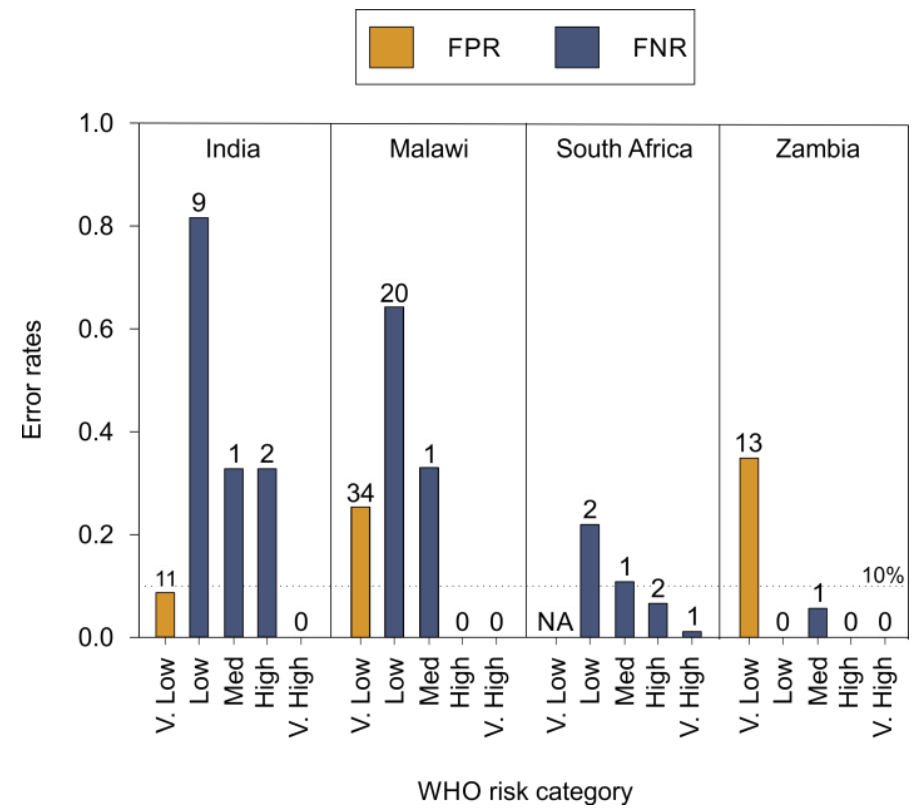

Figure 4. False-positive (FPR) and false-negative (FNR) error rates for presence of TTCs for each WHO risk category in each study using a TLF threshold of $1.3 \mathrm{ppb}$. Number above the bar indicates number of incorrectly classified sites. 


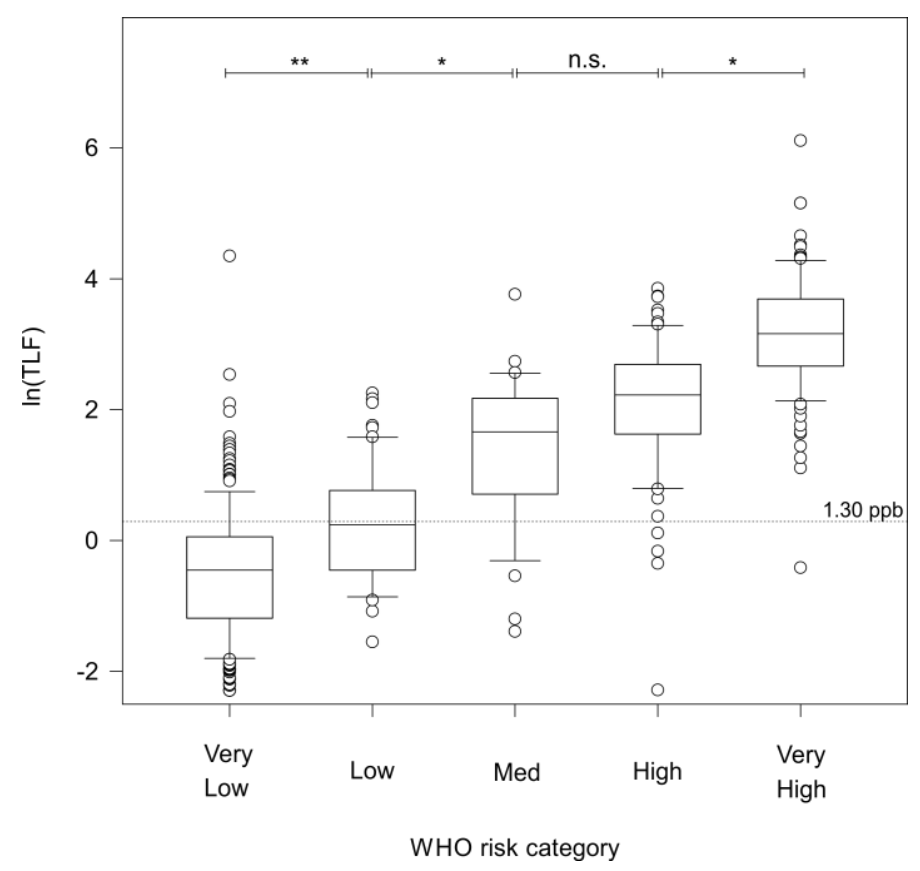

Figure 5. Boxplot of the natural logarithm of tryptophan-like fluorescence by WHO risk category. An addition of $0.2 \mathrm{ppb}$ TLF was made to nine sites to ensure the logarithm could be defined. Significance tests were performed on untransformed TLF data using Dunn's test and are denoted by: not significant $=$ n.s., $\mathrm{p}=0.05(*) \mathrm{p}=0.01\left({ }^{* *}\right)$. Sample sizes: Very Low $=$ 293, Low $=61$, Medium $=32$, High $=71$, Very High $=107$. Boxes illustrate median and interquartile range, whiskers indicate 5th and 95th percentile and all outliers are shown. 


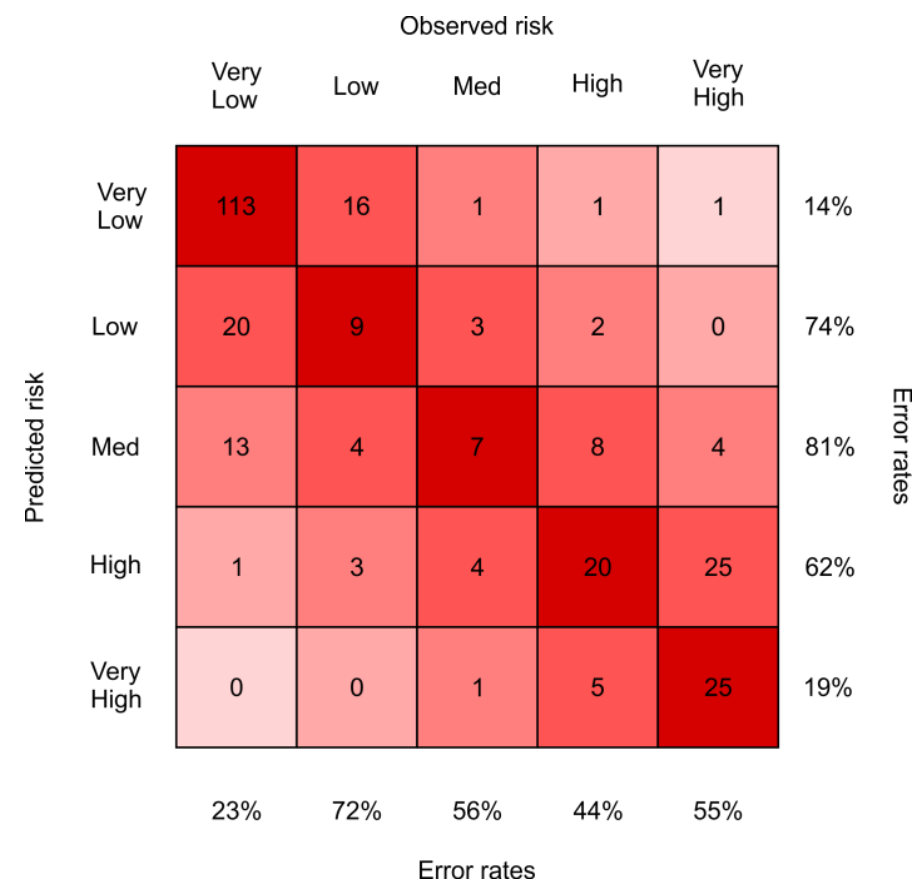

Figure 6. Risk matrix of observed versus predicted risk category.

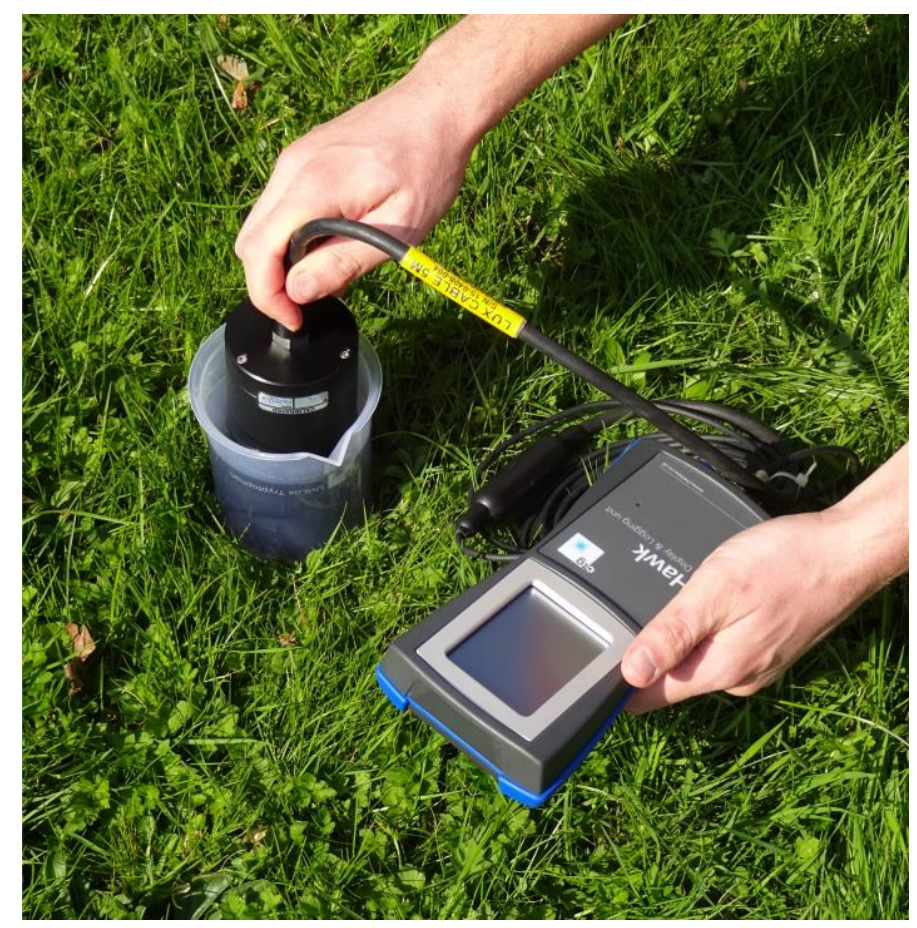

Figure 7. Instantaneous assessment of faecal contamination in drinking water using a tryptophan-like fluorimeter in a beaker and a handheld reader. Note that the sensor operates using UV light and is sensitive to sunlight; hence, the beaker would need to be covered for a reliable measurement. 


\section{Tables}

Table 1. Details of individual TLF-TTC studies.

\begin{tabular}{llllllll}
\hline Study & Source & Fluorimeter & $\begin{array}{l}\text { Faecal } \\
\text { indicator }\end{array}$ & $\begin{array}{l}\text { No. } \\
\text { positive }\end{array}$ & $\begin{array}{l}\text { No. } \\
\text { negative }\end{array}$ & $\begin{array}{l}\text { No. } \\
\text { total }\end{array}$ & Published \\
\hline India & Groundwater & UviLux & TTCs & 45 & 105 & 150 & Sorensen et al. 2016 \\
Malawi & Groundwater & UviLux & TTCs & 49 & 133 & 182 & Unpublished \\
South Africa & Surface water & SMF4 & E. coli & 121 & 0 & 121 & Baker et al. 2015 \\
Zambia & Groundwater & UviLux & TTCs & 74 & 37 & 111 & Sorensen et al. 2015a \\
\hline Total & - & - & - & 289 & 275 & 564 & - \\
\hline
\end{tabular}

Table 2. Summarised UNICEF target product profile for the more rapid detection of E.coli

\begin{tabular}{lll}
\hline Attribute & Minimum performance & Ideal performance \\
\hline Function & Detection of faecal contamination & $\begin{array}{l}\text { Detection of faecal contamination } \\
\text { equivalent to } E \text {. coli }\end{array}$ \\
$\begin{array}{ll}\text { Determining faecal } \\
\text { contamination }\end{array}$ & False-positives $<10 \%$ & False-positives $<5 \%$ \\
Limit of detection & False-negatives $<10 \%$ & False-negatives $<5 \%$ \\
Quantifying faecal contamination & Equivalent to $10 \mathrm{cfu} / 100 \mathrm{~mL}$ & Equivalent to $1 \mathrm{cfu} / 100 \mathrm{~mL}$ \\
& Distinguishes WHO risk categories & Distinguishes equivalent plate count \\
& & \\
Sensor design & Less than $3 \mathrm{~h}$ for result & Less than 30 mins for result \\
& Negative control sterile water & No negative control \\
& Calibration for pH & No calibration \\
& Easy-to-use & Easy-to-use \\
& Battery-powered & Battery-powered \\
& Lightweight and portable & Handheld \\
& 2 year lifespan (hardware) & 5 year lifespan (hardware) \\
& 2 year lifespan (consumables) & 5 year lifespan (consumables) \\
\$1-5 per test (lifetime cost) & \$1-5 per test (lifetime cost) \\
& Turbidity (0-10 NTU) & Turbidity (0-50 NTU) \\
pH (5.5-8.5) & pH (4.5-8.5) \\
salinity (drinking water range) & salinity (drinking water range) \\
\hline
\end{tabular}

Footnote: The WHO risk categories are based on the number of indicator organisms per 100 mL: Very Low risk (0), Low risk (1-9), Medium risk (10-99), High risk (100-999), and Very High risk (1000+) (WHO 1997).

Table 3. Tryptophan-like thresholds for individual risk categories.

\begin{tabular}{ll}
\hline Category & TLF threshold $(\mathrm{ppb})$ \\
\hline Low & 1.3 \\
Medium & 2.4 \\
High & 6.9 \\
Very High & 27.1 \\
\hline
\end{tabular}




\section{Acknowledgements}

This work would not have been possible without the support of Rachel Bell and Ben Marchant (BGS); Steve Sugden and Gopalan Sampath (Water For People), Daniel Nkhuwa, Meki Chirwa, and Joel Kabika (University of Zambia); and Moses Liemisa and Mirriam Chibesa (Lukanga Water and Sewerage Company, Zambia).

James Sorensen, Dan Lapworth, Jade Ward, Alan MacDonald acknowledge support from the UPGro Programme, NERC [NE/002078/1; NE/M008606/1], and Water For People, Canada. Andy Baker acknowledges UK EPSRC [EP/H003061/1].

BGS authors publish with the permission of the Executive Director, British Geological Survey (NERC). Any identification of equipment does not imply recommendation or endorsement by the authors and their respective employers. 


\section{References}

Adler, S., Widerström, M., Lindh, J. and Lilja, M. (2017) Symptoms and risk factors of Cryptosporidium hominis infection in children: data from a large waterborne outbreak in Sweden. Parasitology Research, 1-6.

Arana, I., Seco, C., Epelde, K., Muela, A., Fernández-Astorga, A. and Barcina, I. (2004) Relationships between Escherichia coli cells and the surrounding medium during survival processes. Antonie van Leeuwenhoek 86(2), 189-199.

Baghoth, S., Sharma, S. and Amy, G. (2011) Tracking natural organic matter (NOM) in a drinking water treatment plant using fluorescence excitation-emission matrices and PARAFAC. Water Research 45(2), 797-809.

Bain, R., Cronk, R., Hossain, R., Bonjour, S., Onda, K., Wright, J., Yang, H., Slaymaker, T., Hunter, P. and Prüss-Ustün, A. (2014a) Global assessment of exposure to faecal contamination through drinking water based on a systematic review. Tropical Medicine \& International Health 19(8), 917-927. Bain, R., Cronk, R., Wright, J., Yang, H., Slaymaker, T. and Bartram, J. (2014b) Fecal contamination of drinking-water in low-and middle-income countries: a systematic review and meta-analysis. PLoS medicine 11(5), e1001644.

Baker, A. (2001) Fluorescence excitation-emission matrix characterization of some sewage-impacted rivers. Environmental Science \& Technology 35(5), 948-953.

Baker, A. (2002) Fluorescence properties of some farm wastes: implications for water quality monitoring. Water Research 36(1), 189-195.

Baker, A. (2005) Thermal fluorescence quenching properties of dissolved organic matter. Water Research 39(18), 4405-4412.

Baker, A., Cumberland, S.A., Bradley, C., Buckley, C. and Bridgeman, J. (2015) To what extent can portable fluorescence spectroscopy be used in the real-time assessment of microbial water quality? Science of the Total Environment 532, 14-19.

Baker, A. and Curry, M. (2004) Fluorescence of leachates from three contrasting landfills. Water Research 38(10), 2605-2613.

Baker, A., Elliott, S. and Lead, J.R. (2007) Effects of filtration and pH perturbation on freshwater organic matter fluorescence. Chemosphere 67(10), 2035-2043.

Berg, J.D. and Fiksdal, L. (1988) Rapid detection of total and fecal coliforms in water by enzymatic hydrolysis of 4-methylumbelliferone-beta-D-galactoside. Applied and environmental microbiology 54(8), 2118-2122.

Bridgeman, J., Baker, A., Brown, D. and Boxall, J. (2015) Portable LED fluorescence instrumentation for the rapid assessment of potable water quality. Science of the Total Environment 524, 338-346. Bronk, B.V. and Reinisch, L. (1993) Variability of steady-state bacterial fluorescence with respect to growth conditions. Applied spectroscopy 47(4), 436-440.

Brown, J., Proum, S. and Sobsey, M. (2008) Escherichia coli in household drinking water and diarrheal disease risk: evidence from Cambodia. Water science and technology 58(4), 757-763. Cammack, W.L., Kalff, J., Prairie, Y.T. and Smith, E.M. (2004) Fluorescent dissolved organic matter in lakes: Relationships with heterotrophic metabolism. Limnology and Oceanography 49(6), 2034-2045. Carstea, E.M., Baker, A., Bieroza, M. and Reynolds, D. (2010) Continuous fluorescence excitationemission matrix monitoring of river organic matter. Water Research 44(18), 5356-5366.

Carstea, E.M., Bridgeman, J., Baker, A. and Reynolds, D.M. (2016) Fluorescence spectroscopy for wastewater monitoring: A review. Water Research 95, 205-219.

Chen, J., Alcaine, S.D., Jiang, Z., Rotello, V.M. and Nugen, S.R. (2015) Detection of Escherichia coli in drinking water using 77 bacteriophage-conjugated magnetic probe. Analytical chemistry 87(17), 8977-8984.

Dalterio, R., Nelson, W., Britt, D., Sperry, J., Psaras, D., Tanguay, J. and Suib, S. (1986) Steady-state and decay characteristics of protein tryptophan fluorescence from bacteria. Applied spectroscopy 40(1), 86-90. 
Dalterio, R., Nelson, W., Britt, D., Sperry, J., Tanguay, J. and Suib, S. (1987) The steady-state and decay characteristics of primary fluorescence from live bacteria. Applied spectroscopy 41(2), 234241.

Dartnell, L.R., Roberts, T.A., Moore, G., Ward, J.M. and Muller, J.-P. (2013) Fluorescence characterization of clinically-important bacteria. PloS one 8(9), e75270.

Determann, S., Lobbes, J.M., Reuter, R. and Rullkötter, J. (1998) Ultraviolet fluorescence excitation and emission spectroscopy of marine algae and bacteria. Marine Chemistry 62(1), 137-156.

Dobson, A.J. (2001) An introduction to generalized linear models, CRC press.

Elliott, S., Lead, J. and Baker, A. (2006) Characterisation of the fluorescence from freshwater, planktonic bacteria. Water Research 40(10), 2075-2083.

Fox, B., Thorn, R., Anesio, A. and Reynolds, D. (2017) The in situ bacterial production of fluorescent organic matter; an investigation at a species level. Water Research.

Frahm, E. and Obst, U. (2003) Application of the fluorogenic probe technique (TaqMan PCR) to the detection of Enterococcus spp. and Escherichia coli in water samples. Journal of microbiological methods 52(1), 123-131.

Guion, C.E., Ochoa, T.J., Walker, C.M., Barletta, F. and Cleary, T.G. (2008) Detection of diarrheagenic Escherichia coli by use of melting-curve analysis and real-time multiplex PCR. Journal of clinical microbiology 46(5), 1752-1757.

Gunda, N.S.K., Naicker, S., Shinde, S., Kimbahune, S., Shrivastava, S. and Mitra, S. (2014) Mobile Water Kit (MWK): a smartphone compatible low-cost water monitoring system for rapid detection of total coliform and E. coli. Analytical Methods 6(16), 6236-6246.

Hamilton, W.P., Kim, M. and Thackston, E.L. (2005) Comparison of commercially available Escherichia coli enumeration tests: Implications for attaining water quality standards. Water Research 39(20), 4869-4878.

Hanley, J.A. and McNeil, B.J. (1983) A method of comparing the areas under receiver operating characteristic curves derived from the same cases. Radiology 148(3), 839-843.

Harwood, V.J., Staley, C., Badgley, B.D., Borges, K. and Korajkic, A. (2014) Microbial source tracking markers for detection of fecal contamination in environmental waters: relationships between pathogens and human health outcomes. FEMS microbiology reviews 38(1), 1-40.

Heibati, M., Stedmon, C.A., Stenroth, K., Rauch, S., Toljander, J., Säve-Söderbergh, M. and Murphy, K.R. (2017) Assessment of drinking water quality at the tap using fluorescence spectroscopy. Water Research.

Henderson, R., Baker, A., Murphy, K., Hambly, A., Stuetz, R. and Khan, S. (2009) Fluorescence as a potential monitoring tool for recycled water systems: A review. Water Research 43(4), 863-881. Howard, G., Pedley, S., Barrett, M., Nalubega, M. and Johal, K. (2003) Risk factors contributing to microbiological contamination of shallow groundwater in Kampala, Uganda. Water Research 37(14), 3421-3429.

Hudson, N., Baker, A. and Reynolds, D. (2007) Fluorescence analysis of dissolved organic matter in natural, waste and polluted waters-a review. River Research and Applications 23(6), 631-649. Ikeda, M. (2006) Towards bacterial strains overproducing L-tryptophan and other aromatics by metabolic engineering. Applied microbiology and biotechnology 69(6), 615.

John, D.E. and Rose, J.B. (2005) Review of factors affecting microbial survival in groundwater. Environmental Science \& Technology 39(19), 7345-7356.

Khamis, K., Bradley, C., Stevens, R. and Hannah, D.M. (2016) Continuous field estimation of dissolved organic carbon concentration and biochemical oxygen demand using dual-wavelength fluorescence, turbidity and temperature. Hydrological Processes.

Khamis, K., Sorensen, J., Bradley, C., Hannah, D., Lapworth, D.J. and Stevens, R. (2015) In situ tryptophan-like fluorometers: assessing turbidity and temperature effects for freshwater applications. Environmental Science: Processes \& Impacts 17(4), 740-752. 
Lapworth, D.J., Gooddy, D., Butcher, A. and Morris, B. (2008) Tracing groundwater flow and sources of organic carbon in sandstone aquifers using fluorescence properties of dissolved organic matter (DOM). Applied Geochemistry 23(12), 3384-3390.

Leclerc, H., Mossel, D., Edberg, S. and Struijk, C. (2001) Advances in the bacteriology of the coliform group: their suitability as markers of microbial water safety. Annual Reviews in Microbiology 55(1), 201-234.

$\mathrm{Li}, \mathrm{G}$. and Young, K.D. (2013) Indole production by the tryptophanase TnaA in Escherichia coli is determined by the amount of exogenous tryptophan. Microbiology 159(2), 402-410.

Li, W.-T., Jin, J., Li, Q., Wu, C.-F., Lu, H., Zhou, Q. and Li, A.-M. (2016) Developing LED UV fluorescence sensors for online monitoring DOM and predicting DBPs formation potential during water treatment. Water Research 93, 1-9.

Lopez-Roldan, R., Tusell, P., Cortina, J.L. and Courtois, S. (2013) On-line bacteriological detection in water. TrAC Trends in Analytical Chemistry 44, 46-57.

Maheux, A.F., Bissonnette, L., Boissinot, M., Bernier, J.-L.T., Huppé, V., Picard, F.J., Bérubé, È. and Bergeron, M.G. (2011) Rapid Concentration and Molecular Enrichment Approach for Sensitive Detection of Escherichia coli/Shigella in Potable Water Samples. Applied and environmental microbiology, AEM. 02337-02310.

Moe, C., Sobsey, M., Samsa, G. and Mesolo, V. (1991) Bacterial indicators of risk of diarrhoeal disease from drinking-water in the Philippines. Bulletin of the World Health Organization 69(3), 305. Muller, M., Milori, D.M.B.P., Déléris, S., Steyer, J.-P. and Dudal, Y. (2011) Solid-phase fluorescence spectroscopy to characterize organic wastes. Waste management 31(9), 1916-1923.

Prüss-Ustün, A., Bartram, J., Clasen, T., Colford, J.M., Cumming, O., Curtis, V., Bonjour, S., Dangour, A.D., De France, J. and Fewtrell, L. (2014) Burden of disease from inadequate water, sanitation and hygiene in low-and middle-income settings: a retrospective analysis of data from 145 countries. Tropical Medicine \& International Health 19(8), 894-905.

Radke, S.M. and Alocilja, E.C. (2005) A high density microelectrode array biosensor for detection of E. coli O157: H7. Biosensors and Bioelectronics 20(8), 1662-1667.

Reynolds, D.M. (2003) Rapid and direct determination of tryptophan in water using synchronous fluorescence spectroscopy. Water Research 37(13), 3055-3060.

Rinttilä, T., Kassinen, A., Malinen, E., Krogius, L. and Palva, A. (2004) Development of an extensive set of 16S rDNA-targeted primers for quantification of pathogenic and indigenous bacteria in faecal samples by real-time PCR. Journal of Applied Microbiology 97(6), 1166-1177.

Robin, X., Turck, N., Hainard, A., Tiberti, N., Lisacek, F., Sanchez, J.-C. and Müller, M. (2011) pROC: an open-source package for $\mathrm{R}$ and $\mathrm{S}+$ to analyze and compare ROC curves. BMC bioinformatics 12(1), 77.

Rompré, A., Servais, P., Baudart, J., De-Roubin, M.-R. and Laurent, P. (2002) Detection and enumeration of coliforms in drinking water: current methods and emerging approaches. Journal of microbiological methods 49(1), 31-54.

Seaver, M., Roselle, D.C., Pinto, J.F. and Eversole, J.D. (1998) Absolute emission spectra from Bacillus subtilis and Escherichia coli vegetative cells in solution. Applied optics 37(22), 5344-5347.

Shutova, Y., Baker, A., Bridgeman, J. and Henderson, R. (2016) On-line monitoring of organic matter concentrations and character in drinking water treatment systems using fluorescence spectroscopy. Environmental Science: Water Research \& Technology 2(4), 749-760.

Sohn, M., Himmelsbach, D.S., Barton, F.E. and Fedorka-Cray, P.J. (2009) Fluorescence spectroscopy for rapid detection and classification of bacterial pathogens. Applied spectroscopy 63(11), 12511255.

Sorensen, J., Lapworth, D., Marchant, B., Nkhuwa, D., Pedley, S., Stuart, M., Bell, R., Chirwa, M., Kabika, J. and Liemisa, M. (2015a) In-situ tryptophan-like fluorescence: a real-time indicator of faecal contamination in drinking water supplies. Water Research 81, 38-46. 
Sorensen, J., Lapworth, D., Read, D., Nkhuwa, D., Bell, R., Chibesa, M., Chirwa, M., Kabika, J., Liemisa, M. and Pedley, S. (2015b) Tracing enteric pathogen contamination in sub-Saharan African

groundwater. Science of the Total Environment 538, 888-895.

Sorensen, J., Sadhu, A., Sampath, G., Sugden, S., Gupta, S.D., Lapworth, D., Marchant, B. and Pedley, S. (2016) Are sanitation interventions a threat to drinking water supplies in rural India? An application of tryptophan-like fluorescence. Water Research 88, 923-932.

Stedmon, C.A., Seredyńska-Sobecka, B., Boe-Hansen, R., Le Tallec, N., Waul, C.K. and Arvin, E. (2011) A potential approach for monitoring drinking water quality from groundwater systems using organic matter fluorescence as an early warning for contamination events. Water Research 45(18), 60306038.

UNICEF (2017) Target Product Profile: Rapid E. coli Detection. Version 2.0.

Velasquez-Orta, S., Werner, D., Varia, J. and Mgana, S. (2017) Microbial fuel cells for inexpensive continuous in-situ monitoring of groundwater quality. Water Research 117, 9-17.

WHO (1997) Guidelines for Drinking-Water Quality (2nd Edition) WHO Press, Geneva, Switzerland. WHO (2011) Guidelines for drinking-water quality (4th edition), WHO Press, Geneva, Switzerland. Yamashita, Y. and Tanoue, E. (2003) Chemical characterization of protein-like fluorophores in DOM in relation to aromatic amino acids. Marine Chemistry 82(3), 255-271. 